\title{
Chikungunya virus replication in skeletal muscle cells is required for disease development
}

\author{
Anthony J. Lentscher, ${ }^{1,2}$ Mary K. McCarthy, ${ }^{3}$ Nicholas A. May, ${ }^{3}$ Bennett J. Davenport, ${ }^{3}$ Stephanie A. Montgomery, ${ }^{4}$ \\ Krishnan Raghunathan, ${ }^{2,5}$ Nicole McAllister, ${ }^{1,2}$ Laurie A. Silva, ${ }^{2,5}$ Thomas E. Morrison, ${ }^{3}$ and Terence S. Dermody ${ }^{1,2,5}$ \\ 'Department of Microbiology and Molecular Cenetics, University of Pittsburgh School of Medicine, Pittsburgh, Pennsylvania, USA. ${ }^{2}$ Center for Microbial Pathogenesis, University of Pittsburgh Medical Center \\ Children's Hospital of Pittsburgh, Pittsburgh, Pennsylvania, USA. ${ }^{3}$ Department of Immunology and Microbiology, University of Colorado School of Medicine, Aurora, Colorado, USA. ${ }^{4}$ Department of Pathology \\ and Laboratory Medicine, Lineberger Comprehensive Cancer Center, University of North Carolina at Chapel Hill, Chapel Hill, North Carolina, USA. ${ }^{5}$ Department of Pediatrics, University of Pittsburgh School of \\ Medicine, Pittsburgh, Pennsylvania, USA
}

\begin{abstract}
Chikungunya virus (CHIKV) is an arbovirus capable of causing a severe and often debilitating rheumatic syndrome in humans. CHIKV replicates in a wide variety of cell types in mammals, which has made attributing pathologic outcomes to replication at specific sites difficult. To assess the contribution of CHIKV replication in skeletal muscle cells to pathogenesis, we engineered a CHIKV strain exhibiting restricted replication in these cells via incorporation of target sequences for skeletal muscle cell-specific miR-206. This virus, which we term SKE, displayed diminished replication in skeletal muscle cells in a mouse model of CHIKV disease. Mice infected with SKE developed less severe disease signs, including diminished swelling in the inoculated foot and less necrosis and inflammation in the interosseous muscles. SKE infection was associated with diminished infiltration of T cells into the interosseous muscle as well as decreased production of $/ / 16, / / 6, I p 10$, and Tnfa transcripts. Importantly, blockade of the IL-6 receptor led to diminished swelling of a control CHIKV strain capable of replication in skeletal muscle, reducing swelling to levels observed in mice infected with SKE. These data implicate replication in skeletal muscle cells and release of IL- 6 as important mediators of CHIKV disease.
\end{abstract}

\section{Introduction}

Viral cell and tissue tropism is an important determinant of viral pathogenesis. The capacity to replicate in a particular cell or tissue is often an essential precursor to the development of disease at that site. Following viral replication, damage to cells and tissues can occur directly via the elaboration of cytotoxic viral products or indirectly through induction of harmful host immune responses. Defining the pathologic consequences of replication at specific sites is critical to an understanding of how viruses cause disease. This process is relatively straightforward for viruses with restricted tropism. For example, hepatitis C virus exhibits a highly specific tropism for human hepatocytes, and the resultant hepatitis is attributable to host immune responses in the liver (1). In contrast, it has been challenging to ascribe pathogenic outcomes to replication at discrete sites for viruses displaying broader cell and tissue tropism. This knowledge gap has precluded a precise understanding of molecular mechanisms of pathogenesis for these viruses.

\section{Related Commentary: p. 1099}

Conflict of interest: The authors have declared that no conflict of interest exists. Copyright: ( 2020, American Society for Clinical Investigation. Submitted: April 29, 2019; Accepted: November 26, 2019;

Published: February 10, 2020.

Reference information: J Clin Invest. 2020;130(3):1466-1478.

https://doi.org/10.1172/JCl129893.
Chikungunya virus (CHIKV) is an arthritogenic alphavirus transmitted by Aedes species mosquitoes that displays a broad tissue tropism in mammals (2). Since its discovery and initial characterization in 1952 (3), CHIKV has periodically emerged into human populations to cause outbreaks of disease in subSaharan Africa, where the virus is maintained in an enzootic cycle between mosquitoes and nonhuman primates (4), and in Asia, where the virus is likely maintained in an urban transmission cycle $(5,6)$. More recently, import of the virus into naive populations, including the first recorded cases of local transmission in the Western Hemisphere, has resulted in epidemics affecting millions of individuals $(6,7)$. As many as $90 \%$ of infected persons experience a syndrome characterized by fever, rash, and severe arthralgia and myalgia, with joint disease in many individuals persisting months to years after initial infection $(8,9)$. While the clinical course of CHIKV infection is well-documented, it is not clear how CHIKV causes disease.

Following the bite of a mammalian host by an infected mosquito, CHIKV replicates in fibroblasts, keratinocytes, and macrophages near the site of inoculation (10-12). Virus then disseminates to a variety of sites of secondary replication, including cells of musculoskeletal tissues and the synovium (11, 13-15). Mounting evidence has implicated skeletal muscle as an important site in CHIKV disease development $(14,16,17)$. CHIKV infection in humans results in myalgia in almost all patients (18-22). Biopsies taken from the quadriceps muscle of patients during acute and chronic disease show atrophy and necrosis of muscle fibers (15). 
A $5^{\prime}$

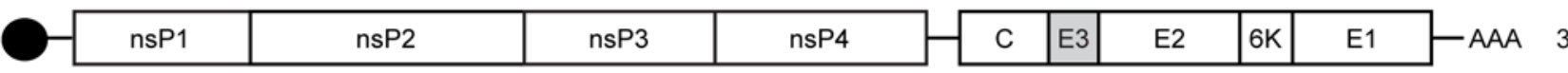

B $5^{\prime}$ \begin{tabular}{l|l|l}
\hline E3 & miR Target 1 \\
\hline
\end{tabular} miR Target 2 miR Target 3 miR Target 4 FMDV 2A Protease

C

$$
\text { E3 }
$$

miR Target 1

miR Target 2

miR Target 3

SKE CCACACACTTCCTTACATTCCACGATCCACACACTTCCTTACATTCCACTCGAGCCACACACTTCCTTACATTCCA

SKE
SKE MM GCCATCCCTCATACATCGTTGCACTCAATACAACATACACTCCCGTATATCCCTCTCGAGCCTCATACATCGTTGCACTCAA miR Target 4

SKE TCACCCACACACTTCCTTACATTCCAAACTTTGACCTACTTAAGTTGGCGGGAGACGTTGAGTCCAACCCTGGGCCC H H H H H H H H H H H H H SKE MM TCACACATACTCTCCCGTATATCCCTAACTTTGACCTACTTAAGTTGGCGGGAGACGTTGAGTCCAACCCTGGGCCCAGTCTT
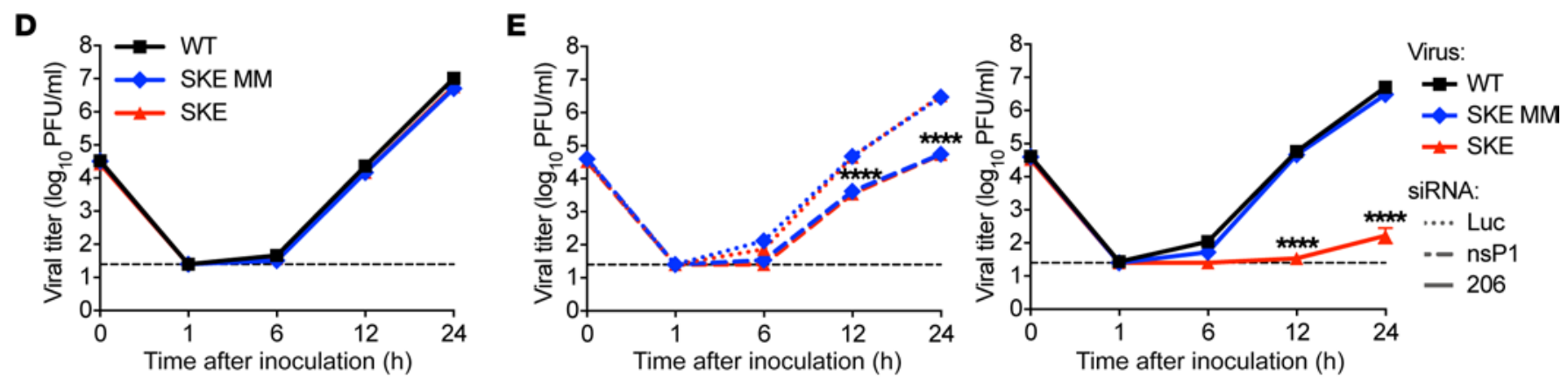

Figure 1. CHIKV engineered to contain target sequences for skeletal muscle-specific miR-206 is specifically restricted by its cognate miRNA. (A) Schematic of the CHIKV genome. Sequences were engineered in-frame within the E3 protein-coding region (shown in grey). (B) Insert cassettes consist of 4 target elements in tandem appended at the $3^{\prime}$ end by sequences of the FMDV 2A protease. (C) Nucleotide sequence of insert cassettes. Four target sequences exhibiting perfect complementarity to skeletal muscle-specific miR-206 were introduced into SKE. Silent mutations were engineered into target sequences to produce SKE mismatch (MM). (D) U-2 OS cells were adsorbed with WT SL15649, SKE MM, or SKE at an MOI of 0.01 PFU/cell. Supernatants were collected at the times shown after adsorption, and viral titer was quantified by plaque assay. (E) U-2 OS cells were transfected with siRNA directed against luciferase (Luc; dotted lines), CHIKV nsP1 (dashed lines), or muscle-specific miR-206-mimic siRNA (solid lines) and adsorbed with WT SL15649 (black), SKE MM (blue), or SKE (red) at an MOI of 0.01 $\mathrm{PFU} /$ cell. Supernatants were collected at the times shown after adsorption, and viral titer was determined by plaque assay. (D and $\mathbf{E})$ Results are expressed as the mean viral titer from duplicate wells of 3 independent experiments. Error bars indicate SEM. Dashed lines indicate the limit of detection. $P$ values were determined at 12 and 24 hpi by ANOVA followed by Tukey's post hoc test. The following comparisons were statistically significant (**** $P<0.0001$ ): SKE MM-Luc versus SKE MM-nsP1 and SKE-Luc versus SKE-nsP1 (E, left), WT-206 versus SKE-206, and SKE MM-206 versus SKE-206 (E, right).

These hallmarks of human infection are recapitulated in a mouse model of CHIKV disease. Mice inoculated with CHIKV in the footpad develop biphasic swelling in the inoculated foot and necrotizing myositis in the interosseous muscles (23). Whether damage caused by CHIKV infection in skeletal muscle is a direct result of viral replication or attributable to elaboration of muscle-injuring inflammatory responses is unknown.

The host RNA silencing machinery can be harnessed to define mechanisms by which viral replication at discrete sites influences pathogenesis. In this pathway, small RNA molecules called miRNAs mediate post-translational silencing of host mRNAs through hybridization to complementary mRNA target sequences (24). An attractive feature of the mammalian RNA silencing pathway is that many miRNAs display cell-typespecific expression (25-27). Therefore, incorporation of target sequences for cell-type-specific miRNAs into the genome of a virus should restrict viral replication specifically in cells expressing the restrictive miRNA while allowing normal replication in all other cell types. This approach was applied to studies of Semliki Forest virus, a related alphavirus, to demonstrate that viral replication in neurons is required for the induction of encephalitis
(28). In this way, the contribution of replication in specific cells and tissues to disease induction can be defined.

Here, we investigated the contribution of CHIKV replication in skeletal muscle cells to disease development. We discovered that a virus restricted in skeletal muscle cells by incorporation of muscle cell-specific miRNA target sequences is attenuated in mice, leading to diminished inflammation and $\mathrm{T}$ cell infiltration into the interosseous muscle near the site of inoculation. Additionally, we provide evidence that replication in skeletal muscle cells promotes release of IL-6, an important mediator of CHIKV-induced inflammation. These findings indicate that replication in skeletal muscle cells is a critical mediator of CHIKV pathogenesis and identify a potential therapeutic target to alleviate symptoms of severe disease.

\section{Results}

Engineering a skeletal muscle-restricted CHIKV variant. CHIKV infection in humans results in a syndrome characterized by severe arthralgia and myalgia (29-31). Replication in skeletal muscle has been associated with development of severe disease $(14,16,17)$, but the pathologic outcome of virus replication in muscle cells has not been well-defined. To understand the contribution of viral 
A
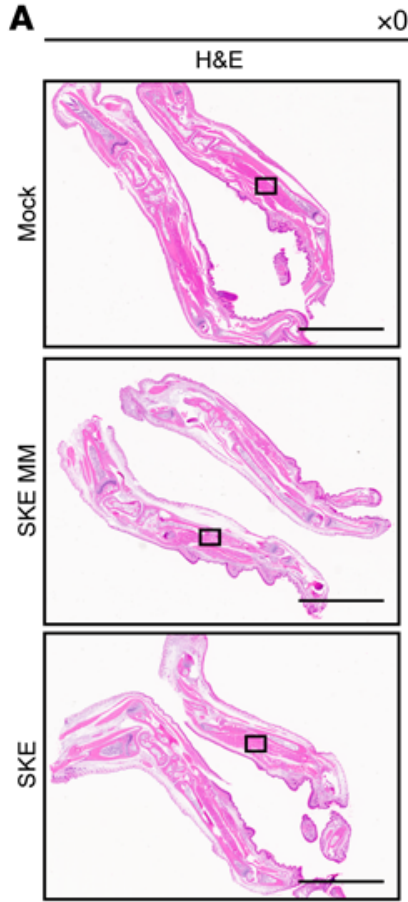

B

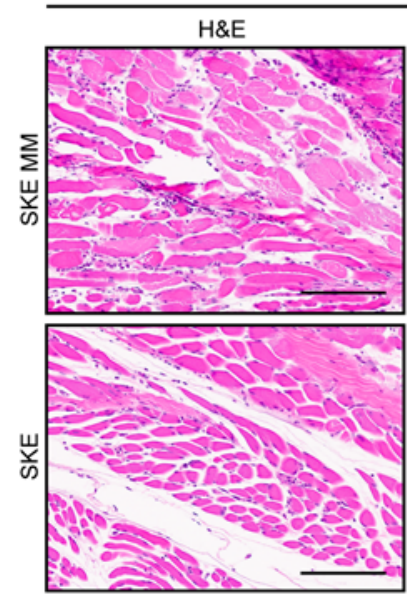

$\times 0.5$
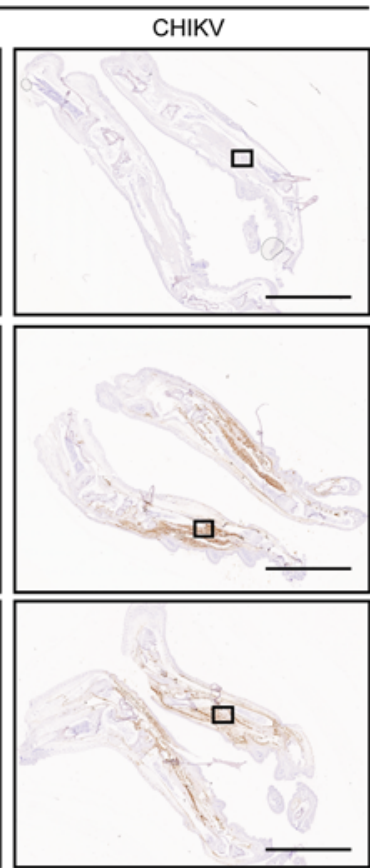

$\times 20$

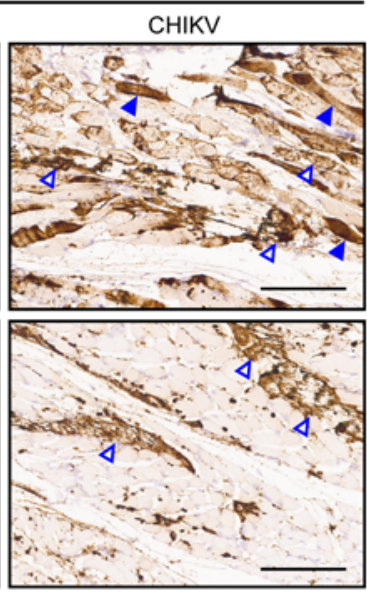

$\times 10$
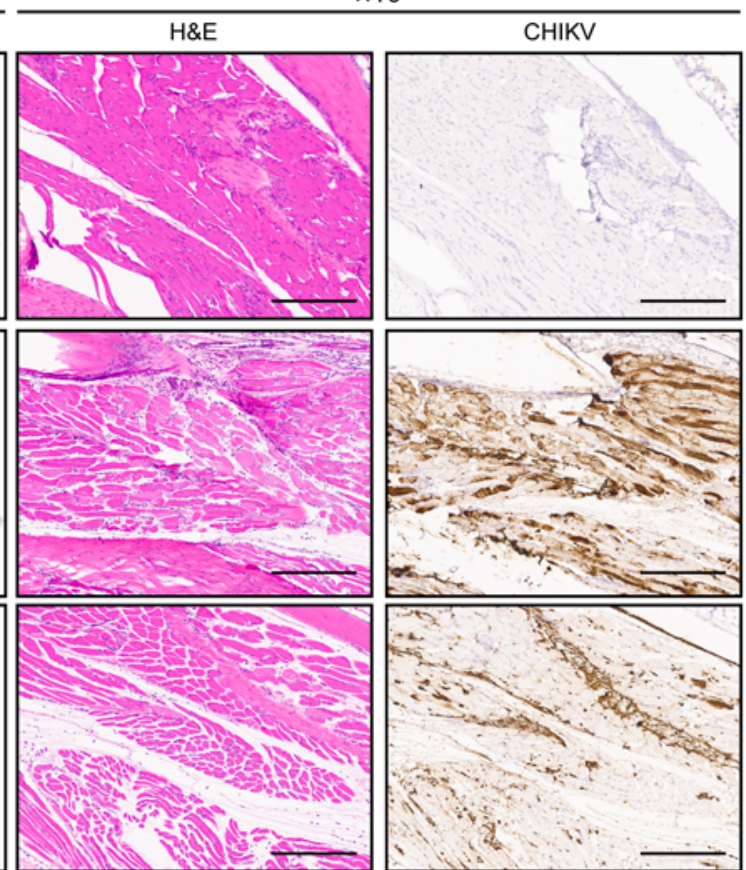

C

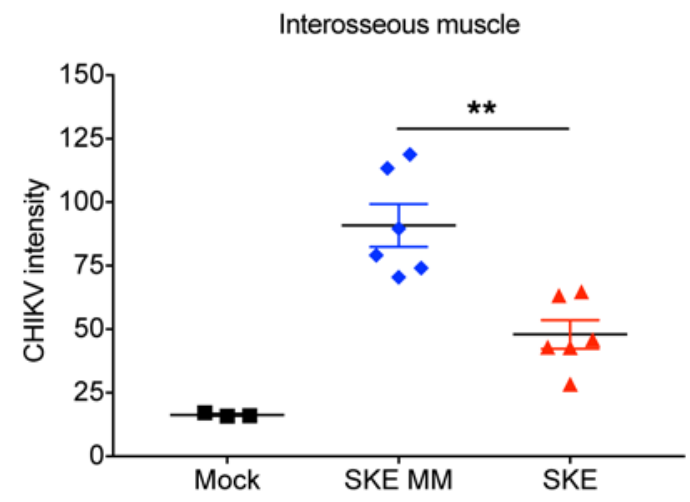

Figure 2. Replication of CHIKV containing muscle-specific miRNA target sequences is restricted in skeletal muscle. Three- to 4-week-old male [57BL/6] mice were inoculated in the left rear footpad with PBS (mock) or $10^{3}$ PFU of SKE MM or SKE. Left ankle tissue was collected 3 days after inoculation and processed for either H\&E staining or RNAscope in situ hybridization for CHIKV RNA. (A) Regions corresponding to high-magnification insets ( $\times 10)$ of the interosseous muscle are indicated in the overview micrographs $(x 0.5)$ by black boxes. Representative images of 3 (mock) or 6 (SKE MM and SKE) mice per group are shown. Scale bars: $\times 0.5,6 \mathrm{~mm} ; \times 10,300 \mu \mathrm{m}$. (B) Magnification insets $\times 20$ with indicated CHIKV staining in myofibers (closed arrows) and connective tissue (open arrows). Scale bars: $\times 20,150 \mu \mathrm{m}$. Images were acquired using an Aperio ScanScope XT slide scanner and processed with Aperio ImageScope software. (C) DAB signal corresponding to CHIKV staining in interosseous muscle was quantified using Imagel software. Horizontal bars indicate mean CHIKV intensity. Error bars indicate SEM. $P$ values were determined comparing SKE and SKE MM by 2 -tailed Student's $t$ test. ${ }^{*} P<0.01$.

replication in skeletal muscle cells to CHIKV disease, we engineered a CHIKV strain exhibiting diminished replication at this site. To achieve this goal, we incorporated into the CHIKV genome target sequences with perfect complementarity to a skeletal muscle cell-specific microRNA, miR-206 $(32,33)$. miR-206 is expressed at detectable levels in skeletal muscle progenitor satellite cells, strongly induced upon differentiation, and then stably expressed at high levels throughout the life of the muscle fiber (34). Target sequences for miR-206 have been employed with other viruses, including coxsackieviruses A21 (35) and B3 (36), to specifically limit repli- cation in myofibers. To ensure stability of the inserted sequences and limit reversion, 4 copies of the miR-206 target sequence were placed in tandem and in-frame into the structural ORF of virulent CHIKV strain SL15649 within coding sequences of the viral E3 glycoprotein (Figure 1, A and B). This site was chosen because it can accommodate insertions of exogenous sequences without compromising replication capacity $(37,38)$. A mismatch control virus was engineered containing silent mutations at synonymous nucleotide positions in miRNA target sequences to alleviate restriction by miR-206 (Figure 1C). Wild-type (WT) SL15649 containing no 
A
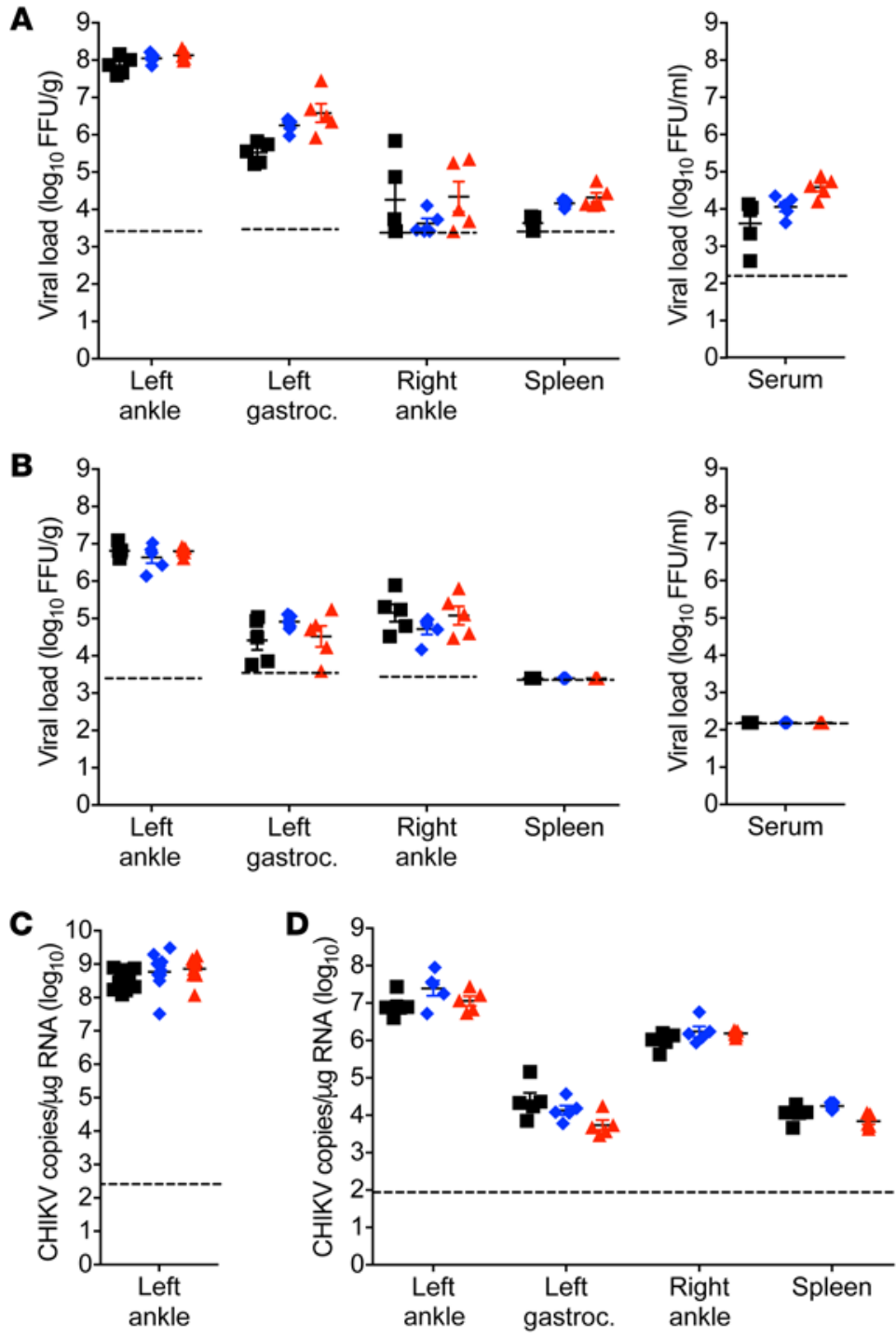

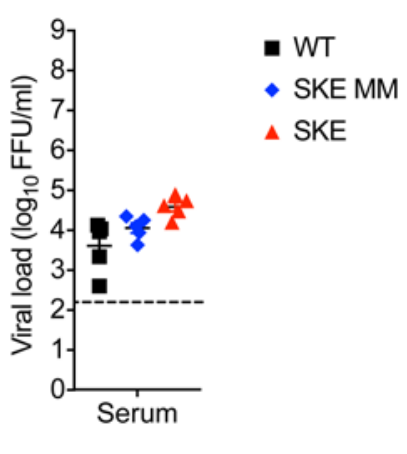

- WT

- SKE MM

$\triangle$ SKE

Figure 3. CHIKV replication in skeletal muscle does not contribute significantly to viral titer in tissue during acute infection. Three- to 4-week-old male [57BL/6] mice were inoculated in the left rear footpad with $10^{3}$ PFU of WT SL15649, SKE MM, or SKE. At 1, 3, and 7 days after inoculation, mice were euthanized; ankles, gastrocnemius (gastroc.) muscles, and spleens were excised; and serum was collected. Viral titers in day 1 (A) and 3 (B) tissue homogenates and serum were determined by FFU assay. Horizontal bars indicate mean $\mathrm{FFU} / \mathrm{g}$ (tissue) or $\mathrm{FFU} / \mathrm{mL}$ (serum) for 5 mice per group. Viral loads in day 3 (C) and 7 (D) tissue homogenates were determined by RT-qPCR. Horizontal bars indicate mean CHIKV genome copies/ $\mu \mathrm{g}$ RNA for 5 (7 dpi) or 10 (3 dpi) mice per group. Error bars indicate SEM. Dashed lines indicate the limits of detection. $P$ values were determined by comparing SKE and SKE MM by ANOVA followed by Tukey's post hoc test. None of the differences are statistically significant.

To determine susceptibility of SKE and SKE MM to miRNA-mediated restriction, a multistep replication experiment was conducted using U-2 OS cells transfected with various siRNAs. Both SKE and SKE MM replicated with kinetics comparable to WT CHIKV in cells transfected with a nontargeting siRNA

- WT

- SKE MM

$\triangle$ SKE directed against luciferase (Figure 1E). Cells transfected with an siRNA targeting the viral nsP1 gene allowed diminished but equivalent replication of SKE and SKE MM, indicating that both strains are equally susceptible to nsP1 siRNA-mediated restriction (Figure 1E). In cells transfected with an siRNA mimicking the sequence of miR-206, replication of SKE was restricted, reaching peak titers significantly lower than those produced by SKE MM and WT CHIKV, which replicated with similar kinetics and produced equivalent peak titers (Figure

miRNA target insertion, skeletal muscle-restricted (SKE), and mismatch control (SKE MM) CHIKV strains were recovered following electroporation of in vitro transcribed viral RNA into baby hamster kidney (BHK-21) cells. Consensus sequencing of SKE and SKE MM stocks harvested at 48 hours after electroporation confirmed maintenance of inserted sequences.

SKE is restricted by cognate miR-206 in vitro. Replication kinetics of SKE and SKE MM were first assessed using nonrestrictive conditions to ensure that no defects in replication were conferred by incorporation of exogenous sequences into the structural ORF of the viral genome. Human osteosarcoma (U-2 OS) cells, which do not naturally express miR-206, were infected with WT CHIKV, SKE, or SKE MM at an MOI of 0.01 $\mathrm{PFU} /$ cell, and supernatants were harvested at various intervals after adsorption to quantify viral progeny production by plaque assay. SKE and SKE MM replicated with kinetics comparable to WT CHIKV and reached similar peak titers in U-2 OS cells (Figure 1D). These data indicate that sequences inserted into the $\mathrm{E} 3$ coding region do not compromise $\mathrm{CHIKV}$ replication in $\mathrm{U}-2$ OS cells.
$1 \mathrm{E})$. These data demonstrate that SKE is specifically restricted by its cognate miRNA in cell culture and that mismatch mutations present in SKE MM are sufficient to alleviate this restriction.

SKE displays diminished replication in murine skeletal muscle. To assess whether SKE is restricted in skeletal muscle tissue in vivo, 3- to 4-week-old C57BL/6J mice were inoculated in the left rear footpad with $10^{3} \mathrm{PFU}$ of either SKE or SKE MM. At day 3 after inoculation, mice were euthanized and the left rear limb was processed for histology. Myofibers were identified in H\&Estained sections as striated, multinucleated cells containing nuclei at the cell periphery (Figure 2, A and B). CHIKV replication in skeletal muscle was assessed by in situ hybridization of serial tissue sections using a probe specific for CHIKV RNA. In mice infected with SKE MM, abundant staining was observed in the interosseous muscles of the foot (Figure 2, A and B), which is consistent with prior studies with WT CHIKV (14). This staining was significantly reduced in mice infected with SKE (Figure 2C), demonstrating that SKE replication is restricted in skeletal muscle cells in mice. Importantly, intense staining was observed in connective tissue of mice infected with either SKE or SKE MM 


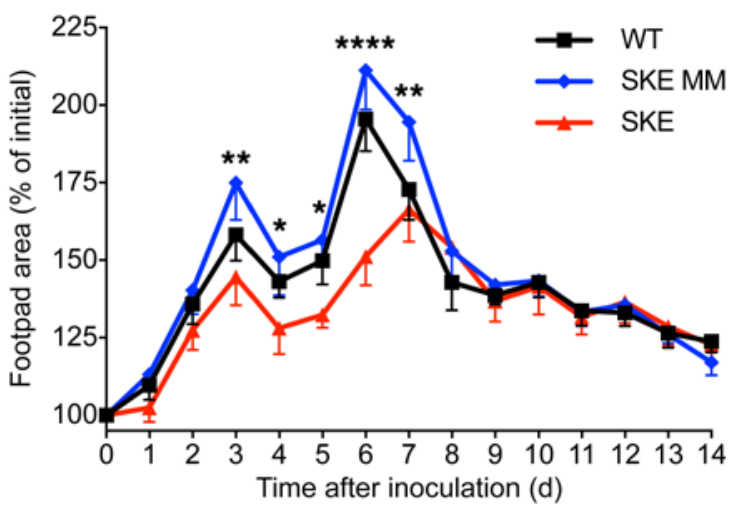

(Figure 2B), indicating that incorporation of target sequences for miR-206 into SKE specifically restricts replication in skeletal muscle cells while still allowing replication at other sites.

SKE and SKE MM produce comparable titers in musculoskeletal tissue of the inoculated foot in mice. Because SKE replication is restricted in skeletal muscle cells, we assessed whether replication in these cells contributes to overall viral titers in foot tissue and viral dissemination during acute infection. Mice were inoculated with WT CHIKV, SKE, or SKE MM, and viral burdens in various tissues both proximal and distal to the site of inoculation were quantified at days 1, 3, and 7 after inoculation. Because the level of infectious virus at day 7 after inoculation is often at or below the limit of detection in our infectivity assays, we used a focus-forming unit (FFU) assay to quantify infectious virus at days 1 and 3 after inoculation and real-time reverse transcription PCR (RT-qPCR) to quantify viral genome copies at day 7 after inoculation. Tissue burdens of SKE MM and SKE did not differ significantly in the left ankle near the site of inoculation or at sites of dissemination, including the left gastrocnemius muscle, right ankle, and spleen, at days 1 (Figure 3A), 3 (Figure 3B), or 7 (Figure 3D) after inoculation. At all time points examined, both viruses produced titers comparable to those produced by WT CHIKV. CHIKV RNA in the interosseous muscle of the left rear foot was significantly reduced at day 3 after inoculation in our in situ hybridization analysis (Figure 2C). Because total viral titers in the ankle did not differ between mice infected with SKE and SKE MM at this time point by FFU assay (Figure 3B), we also analyzed total ankle homogenates by RT-qPCR. This analysis confirmed that, while SKE replication is specifically restricted in interosseous muscle, total viral RNA levels in the ankle produced by SKE and SKE MM at this time point are comparable (Figure 3C). These data indicate that replication in skeletal muscle does not contribute significantly to overall CHIKV titers in tissues or dissemination during acute infection and that replication in other cell types, likely connective tissue fibroblasts that are highly susceptible for CHIKV infection, is responsible for high viral titers observed in musculoskeletal tissues at these time points. Additionally, titers of SKE and SKE MM reached similar levels in the serum at day 1 after inoculation (Figure 3A) and were cleared by day 3 after inoculation (Figure $3 \mathrm{~B}$ ), indicating that restriction of CHIKV replication in skeletal muscle cells does not affect establishment or clearance of viremia.

Restriction of CHIKV replication in skeletal muscle cells attenuates viral virulence. To define the contribution of viral replication in myofibers to pathogenesis, mice were inoculated with WT
Figure 4. Restriction of CHIKV replication in muscle diminishes footpad inflammation. Three- to 4-week-old male [57BL/6] mice were inoculated in the left rear footpad with PBS (mock) or $10^{3}$ PFU of WT SL15649, SKE MM, or SKE. Left rear footpad swelling was quantified using digital calipers on the days shown. Results are normalized to initial footpad area and presented as the mean percentage of initial footpad area for 10 mice per group. Error bars indicate SEM. $P$ values were determined by comparing SKE and SKE MM by ANOVA followed by Tukey's post hoc test. ${ }^{*} P<0.05$; ${ }^{* *} P<0.01 ;{ }^{* * * *} P<0.0001$

CHIKV, SKE, or SKE MM, and swelling of the inoculated foot, a hallmark of CHIKV-mediated disease in mice (23), was measured using digital calipers. Following inoculation with either WT CHIKV or SKE MM, mice exhibited a bimodal pattern of swelling, with swelling peaking at days 3 and 6 after inoculation (Figure $4)$, which is consistent with prior studies $(39,40)$. Mice infected with SKE displayed significantly diminished swelling during both phases, with the second phase peaking later at day 7 after inoculation (Figure 4). These data implicate skeletal muscle cells as a site of viral replication essential to the induction of both phases of swelling following CHIKV infection in mice.

To understand how CHIKV replication in skeletal muscle cells influences tissue injury, mice were inoculated with SKE or SKE MM, euthanized at day 7 after inoculation, and the left rear limb was processed for histology. H\&E-stained tissue sections were scored by a veterinary pathologist blinded to the conditions of the experiment to compare severity of synovitis, inflammation and necrosis in the interosseous muscle, and tenosynovitis. SKE MM-infected mice displayed significant myositis and necrosis of the interosseous muscle, with most mice displaying greater than $40 \%$ displacement of muscle tissue with infiltrating leukocytes (Figure 5A). This finding is consistent with prior studies with WT CHIKV (23). Synovitis was reduced in SKE-infected mice compared with those infected with SKE MM, although scores did not differ significantly (Figure 5B). In contrast, mice infected with SKE exhibited significantly diminished necrosis and inflammation in the interosseous muscle as well as diminished tenosynovitis in the tendon sheath compared with SKE MM-infected mice (Figure 5B). These findings suggest that while replication in skeletal muscle cells does not contribute significantly to tissue viral burden, these cells are an important site of replication for development of muscle inflammation and necrosis.

Diminished CHIKV replication in skeletal muscle cells results in decreased infiltration of $T$ cells into interosseous muscle. $\mathrm{CD}^{+} \mathrm{T}$ cells are important mediators of inflammation and disease following CHIKV infection in mice (39). To determine how CHIKV replication in skeletal muscle cells affects recruitment of T cells into musculoskeletal tissues, mice were inoculated with SKE or SKE MM and euthanized at day 7 after inoculation. The left rear limb was processed for immunohistochemistry using an antibody directed against CD3 (Figure 6A) and stained with H\&E (Figure 6B). Mice infected with SKE exhibited significantly diminished infiltration of $\mathrm{CD}^{+}$cells into the interosseous muscle relative to mice infected 
A
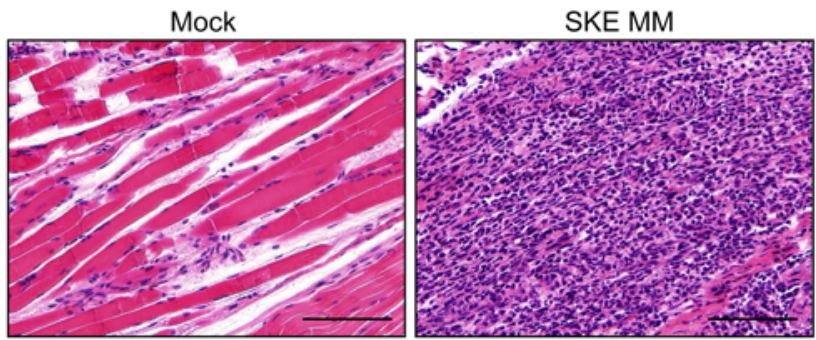

Synovitis

B

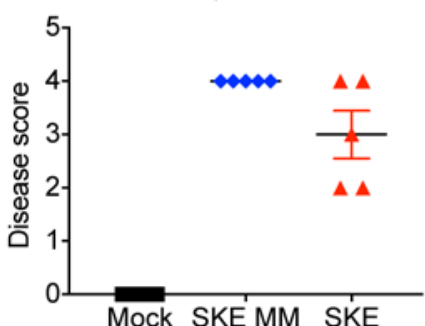

Interosseous muscle

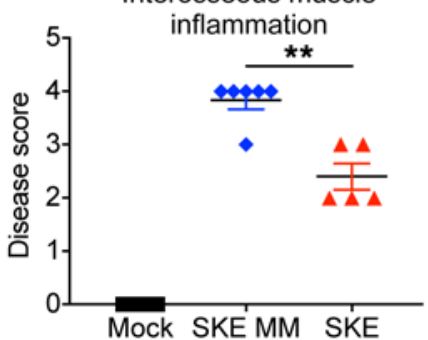

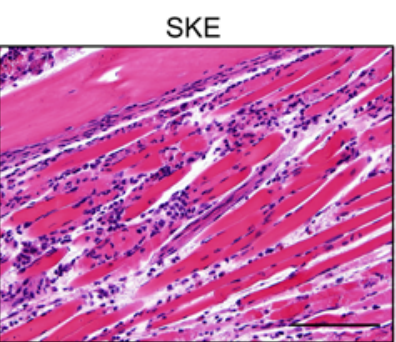

Interosseous muscle

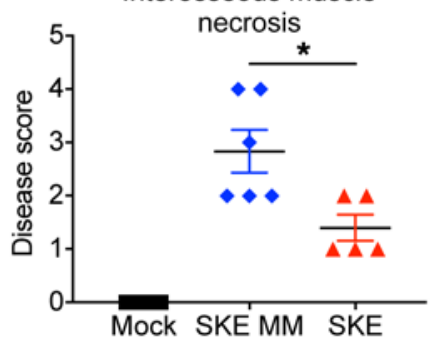

Tenosynovitis

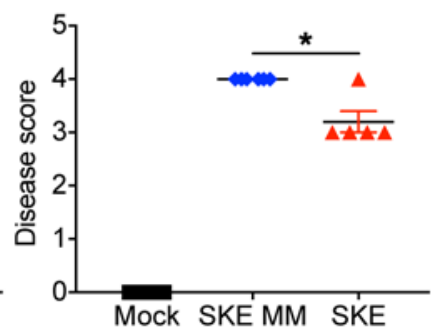

Figure 5. Restriction of CHIKV replication in skeletal muscle diminishes interosseous muscle inflammation and necrosis. Three- to 4-week-old male C57BL/6) mice inoculated in the left rear footpad with PBS (mock) or $10^{3}$ PFU of WT SL15649, SKE MM, or SKE. (A) H\&E staining of left rear interosseous muscle of mock-, SKE MM-, or SKE-infected mice 7 days after inoculation. Data are representative of 2 independent experiments with 3 mice per group in each experiment. Scale bars: $100 \mu \mathrm{m}$. (B) H\&E-stained sections were scored for histological evidence of synovitis, inflammation, and necrosis in the interosseous muscle, and tenosynovitis. Results are expressed as disease score of tissues from individual animals for 5 to 6 mice per group. Horizontal bars indicate mean disease score. Scores were assigned based on the following scale: 0 , no lesions; 1 , mild, fewer than 5 areas of small clusters of leukocytes; 2 , moderate, leukocytes forming larger clusters to thin tracts throughout the tissue, multiple sites/tissues affected; 3 , severe, clusters and tracts of leukocytes coalescing into at least 1 large area that displaces/replaces tissue; 4, markedly severe, leukocytes in aggregates sufficient to replace more than $40 \%$ of normal tissue. Error bars indicate SEM. $P$ values were determined by comparing SKE and SKE MM by Mann Whitney test. ${ }^{*} P<0.05$; ${ }^{* *} P<0.01$.

with SKE MM (Figure 6, A and C). This reduction in infiltrating $\mathrm{CD}^{+} \mathrm{T}$ cells appears to be specific for the interosseous muscle, as the calcaneal tendon, a representative connective tissue, of both SKE- and SKE MM-infected mice was heavily infiltrated with these cells compared with mock-infected mice. These results were reproduced when immunohistochemistry was conducted using an antibody to CD4 (Figure 6D), although staining was less intense. Overall, these data indicate that replication in skeletal muscle cells is a required precursor to $\mathrm{T}$ cell infiltration into this site.

CHIKV replication in skeletal muscle cells is important for production of proinflammatory mediators. We next tested whether diminished foot swelling and pathology following infection with SKE is attributable to altered immune responses elicited by virus incapable of replicating in skeletal muscle cells. Severe CHIKV disease in humans is associated with the production of IL-6, RANTES, and TNFA, with levels of IFNG, IL-1B, IP10, MCP1, and MIP1A also increasing during infection (19, 41, 42). To understand how replication in myofibers influences production of these proinflammatory mediators, mice were infected with SKE or SKE MM, and proinflammatory mediator induction in the left rear foot at day 3 after inoculation was quantified by RT-qPCR. Relative to mice infected with SKE MM, mice infected with SKE exhibited a significant reduction in Il1b, Il6, Ip1O, and Tnfa transcripts in the left rear foot (Figure 7A). Importantly, this reduction was not due to a global decrease in the transcript levels of inflammatory molecules, as mRNA levels of Ifng, Mcp1, Mip1a, and Rantes did not differ significantly in mice infected with SKE compared with SKE MM (Figure 7A). Additionally, while levels of $I l 1 b, I l 6, I p 10$, and Tnfa transcripts tended to be higher following infection with SKE MM, induction of these proinflammatory mediators did not differ significantly in the contralateral foot (Figure 7B), which does not swell following infection with either SKE or SKE MM (data not shown). These data suggest that local production of specific proinflammatory mediators drives swelling of the inoculated foot during CHIKV infection in mice.

IL-6 produced following viral replication in skeletal muscle mediates CHIKV-induced inflammation. Because increased IL-6 production is associated with severe CHIKV disease and production of IL-6 is dependent on virus replication in skeletal muscle cells (Figure 7), we next assessed the contribution of IL-6 to CHIKV pathogenesis. Mice were inoculated intraperitoneally with $200 \mu \mathrm{g}$ of either an IL-6 receptor blocking antibody or an IgG2b isotype control at 0,3 , and 5 days after inoculation with either SKE or SKE MM. Swelling of the inoculated foot was quantified using digital calipers. As expected, SKE MM-infected mice treated with the isotype control exhibited significant swelling that peaked 6 days after inoculation (Figure 8A). In contrast, swelling in SKE MMinfected mice treated with the IL- 6 receptor blockade antibody was significantly reduced 5,6 , and 7 days after inoculation, with levels more comparable to the swelling induced in SKE-infected mice treated with the isotype control antibody (Figure 8A). This phenotype was not due to differences in viral replication, as viral loads in the left and right ankles of SKE MM-infected mice treated with either the IL-6 receptor antibody or isotype control were comparable at day 7 after inoculation (Figure 8B). Thus, IL-6 released following $\mathrm{CHIKV}$ infection in skeletal muscle cells is a critical mediator of CHIKV disease in mice. 
A

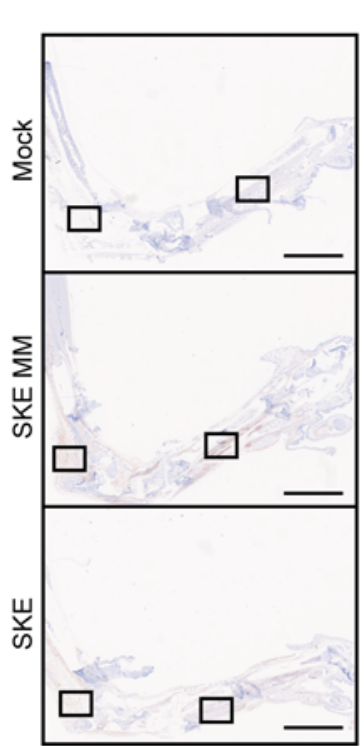

B

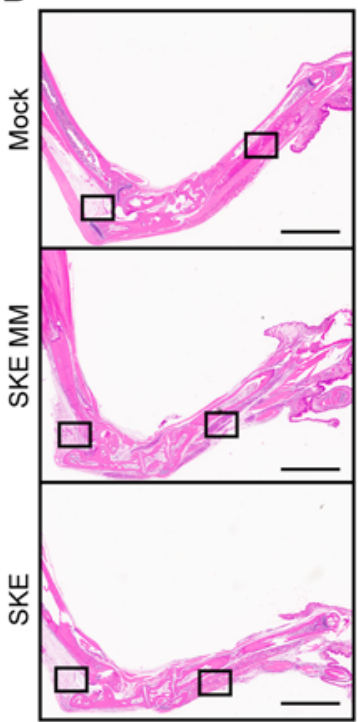

$\times 10$
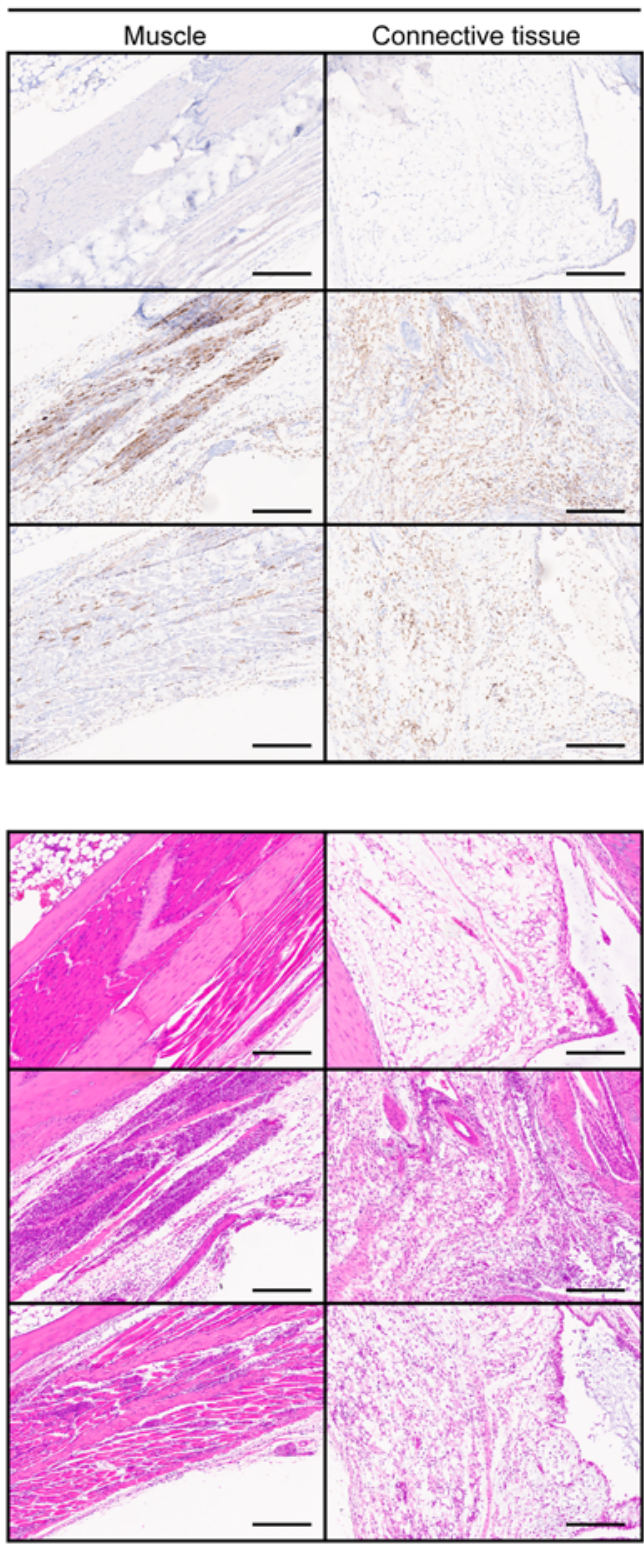

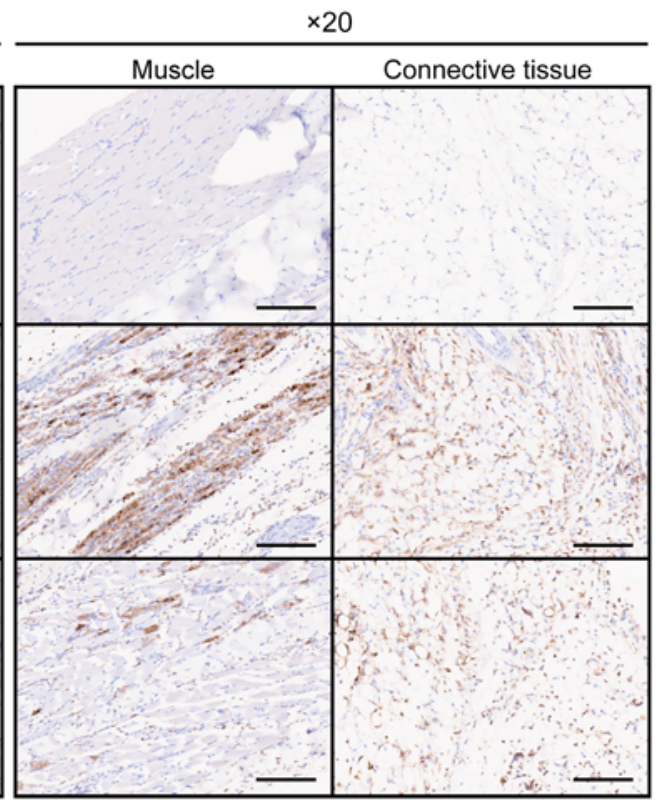

C

Interosseous muscle

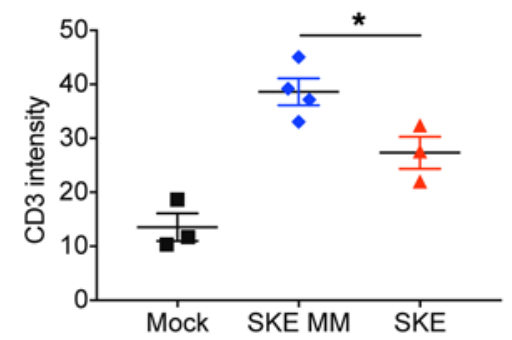

D

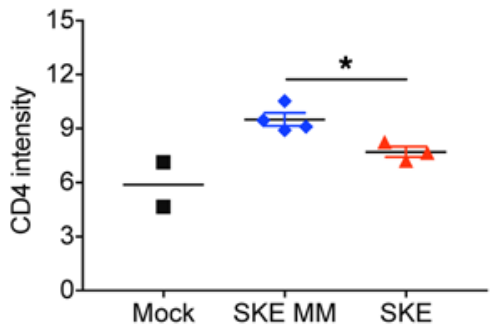

Figure 6. CHIKV replication in myofibers promotes T cell infiltration into interosseous muscle. Three- to 4-week-old male [57BL/6] mice were inoculated in the left rear footpad with PBS (mock) or $10^{3}$ PFU of either SKE MM or SKE. Left ankle tissue was collected 7 days after inoculation and processed for either CD3 immunohistochemistry (A) or H\&E staining (B). Regions corresponding to high-magnification insets $(\times 10)$ of the interosseous muscle and calcaneal tendon (connective tissue) are indicated in the overview micrographs ( $\times 1$ ) by black boxes. Representative images of 3 (mock and SKE) or 4 (SKE MM) mice per group are shown. Scale bars: $\times 1,3 \mathrm{~mm} ; \times 10,300 \mu \mathrm{m} ; \times 20,150 \mu \mathrm{m}$. Images were acquired using an Aperio ScanScope XT slide scanner and processed with Aperio ImageScope software. DAB signal corresponding to $\mathrm{CD} 3$ (C) or CD4 (D) staining in interosseous muscle was quantified using Imagej software. Horizontal bars indicate mean intensity. Error bars indicate SEM. $P$ values were determined comparing SKE and SKE MM by 2-tailed Student's $t$ test. ${ }^{*} P<0.05$.

\section{Discussion}

The broad tropism of CHIKV has made it challenging to define determinants of CHIKV pathogenesis. In this study, we exploited the host RNAi machinery to assess how CHIKV replication in skeletal muscle cells influences disease development. Target sequences for a skeletal muscle cell-specific miRNA, miR-206, were engineered into the structural ORF of the CHIKV genome. The presence of these target sequences was sufficient to specifically restrict CHIKV replication in a miR-206-dependent manner in vitro. Additionally, viral replication was diminished in the interosseous muscles of infected 3- to 4-week-old mice following footpad inoculation with CHIKV, as assessed by in situ hybridization for CHIKV RNA. Interestingly, restriction of replication in skeletal muscle cells did not significantly affect CHIKV titers in tissues, implicating other cell types, likely connective tissue fibroblasts (13), as the major contributor to viral loads in tissues. Restriction of CHIKV replication in skeletal muscle cells led to significantly reduced inflammation and necrosis in the interosseous muscles of the left foot, with a concomitant decrease in $\mathrm{T}$ cell infiltration and transcript levels of select proinflammatory 
A
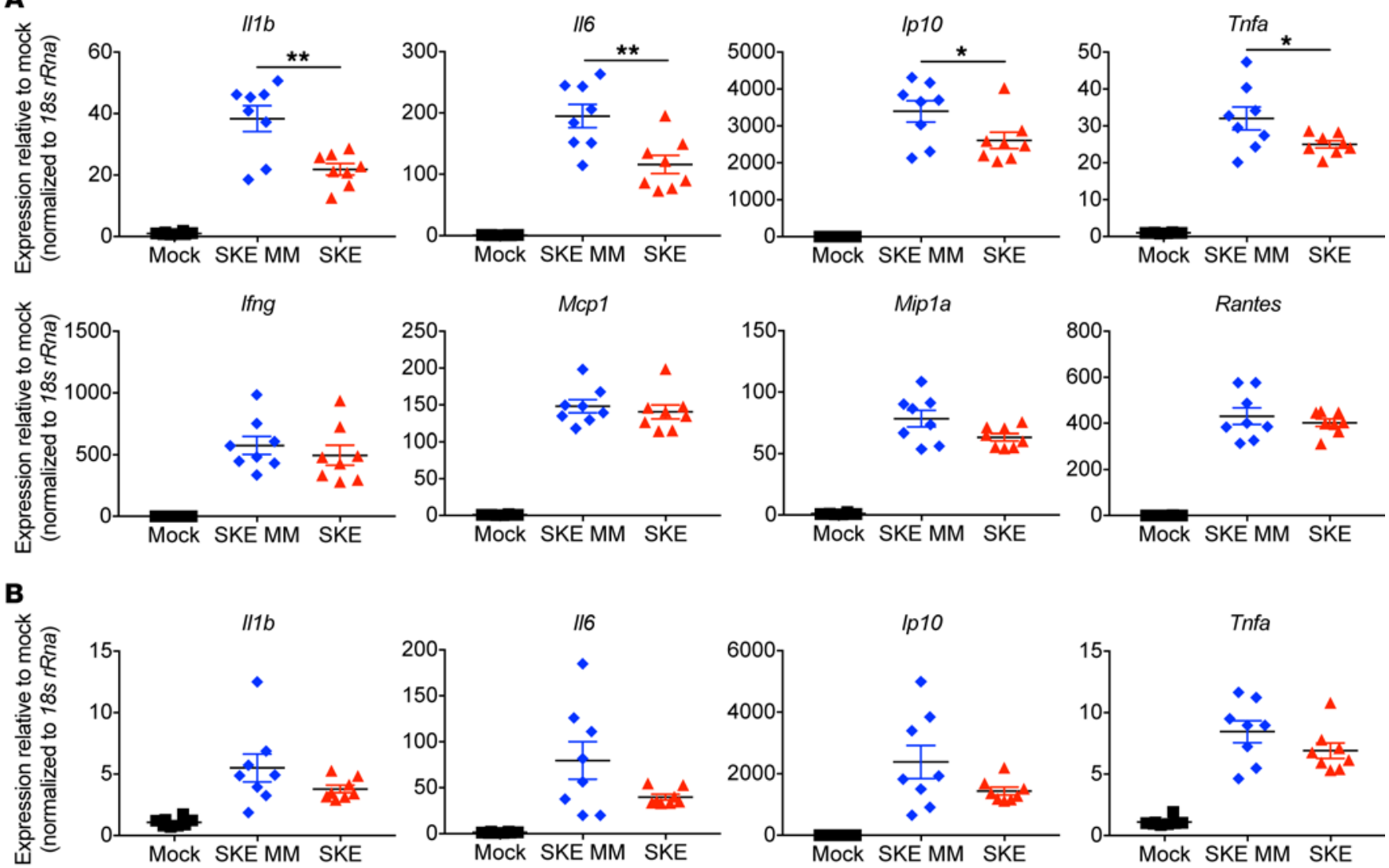

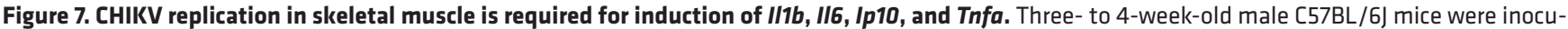
lated in the left rear footpad with PBS (mock) or $10^{3}$ PFU of either SKE MM or SKE. At 3 days after inoculation, mice were euthanized, and left (A) and right (B) ankles were excised. RNA was extracted and reverse transcribed, and expression of the genes shown was quantified by qPCR. Data are normalized to $18 \mathrm{~s} r R n a$ levels and expressed as the relative expression ( $n$-fold increase) over that in mock-infected tissue. Horizontal bars indicate means of 2 independent experiments with 4 mice per group in each experiment. Error bars indicate SEM. $P$ values were determined by comparing SKE and SKE MM by 2-tailed Student's $t$ test. ${ }^{*} P<0.05 ;{ }^{* *} P<0.01$.

mediators including Il1b, Il6, Ip10, and Tnfa. Finally, we found that IL-6 released following CHIKV replication in skeletal muscle cells is an important mediator of inflammation. Accordingly, treatment with an IL-6 receptor antibody significantly diminished swelling in the inoculated foot of mice infected with a control virus capable of replication in skeletal muscle cells to levels similar to those produced by virus restricted at this site.

The capacity to replicate in skeletal muscle is common among arthritogenic alphaviruses and other viruses that cause myalgia and myositis $(43,44)$. Whether these viruses cause disease in skeletal muscle using mechanisms similar to CHIKV is unknown. Importantly, it is unclear even for the leading causes of viral myositis, including enteroviruses and influenza virus, whether virus replication in muscle cells directly damages muscle tissue or if disease results from immunologic processes induced by viral infection (45-47). While CHIKV has primarily been studied in the context of disease manifestations in the joints, CHIKV-patient biopsies demonstrate that virus also elicits significant infection and damage in muscle tissue (15), a facet of disease that was previously underappreciated. Additionally, intradermal inoculation of newborn mice with a virulent strain of CHIKV isolated in Senegal in 1983 results in diminished hind limb weakness and viral titer in muscle compared with inoculation of a more contemporary strain isolated in La Reunion in 2006, implicating viral replication in skeletal muscle as an important, and perhaps strain-specific, mediator of pathogenesis (16). Unfortunately, the divergent nature of these strains has precluded a precise determination of the exact mechanism of attenuation. Understanding how CHIKV causes damage to skeletal muscle is important both to gain a broader understanding of mechanisms of CHIKV pathogenesis and to inform studies defining mechanisms by which other viruses cause muscle pathology.

Restriction of CHIKV replication in skeletal muscle resulted in diminished induction of $I l 1 b, I l 6, I p 10$, and Tnfa in mice. Of these, IL-1B and IL- 6 are biomarkers of severe CHIKV disease in humans (41), although IP10 and TNFA also are significantly induced following CHIKV infection $(19,42)$. The cellular source of these cytokines during infection is unknown. Although produced by a variety of cells (including activated macrophages and T cells), IL-1B, IL-6, IP10, and TNFA could conceivably be directly elaborated by skeletal muscle cells following infection. Skeletal muscle is capable of producing IL-6 and IP10 following contraction of muscle fibers, which is important in muscle regeneration and homeostasis (48-52). Additionally, muscle injury results 

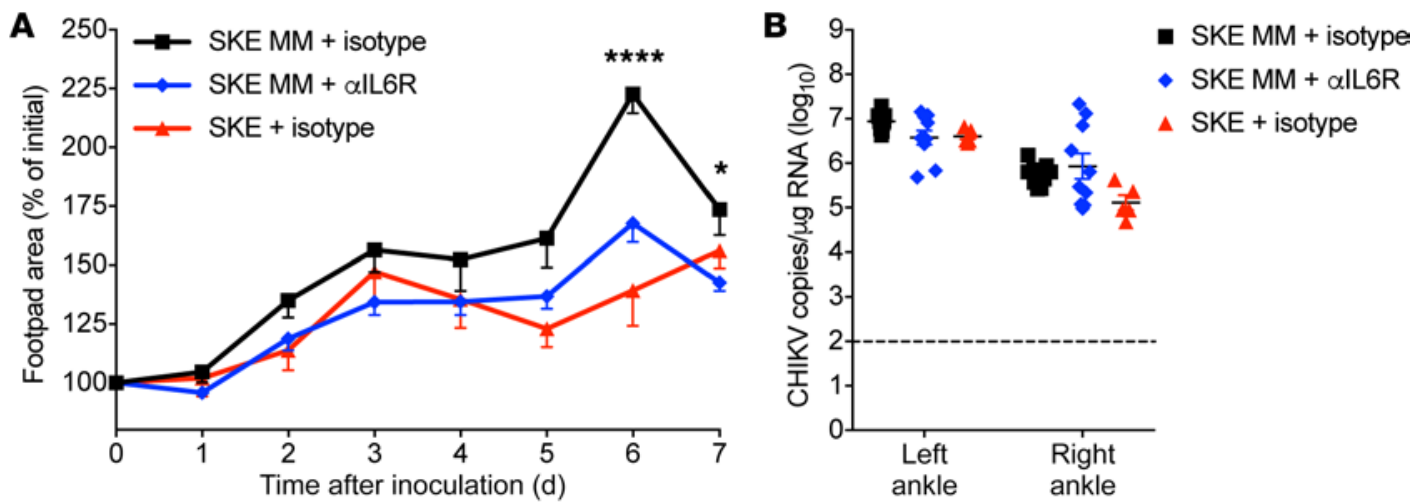

Figure 8. IL-6 mediates CHIKV-induced inflammation. Three- to 4-week-old male C57BL/6] mice were inoculated intraperitoneally with $200 \mu \mathrm{g}$ of either a monoclonal anti-IL-6 receptor antibody (clone 15A7) or an IgG2b isotype control antibody (clone LTF-2) on days 0,3 , and 5 after inoculation with $10^{3}$ PFU of either SKE MM or SKE. (A) Swelling of the left rear footpad was quantified using digital calipers on the days shown. Results are normalized to initial footpad area and presented as the mean percentage of initial footpad area for 10 mice per group (SKE MM + isotype and SKE MM + alL6R) or 5 mice per group (SKE + isotype). Error bars indicate SEM. $P$ values were determined by comparing SKE MM-infected animals receiving IL6R antibody or isotype control by ANOVA followed by Tukey's post hoc test. ${ }^{*} P<0.05$; ${ }^{* * *} P<0.0001$. (B) Viral loads in day 7 tissue homogenates were determined by RT-qPCR. Horizontal bars indicate mean CHIKV genome copies/ $\mu$ g RNA of 10 mice per group (SKE MM + isotype and SKE MM + alL6R) or 5 mice per group (SKE + isotype). Error bars indicate SEM. Dashed line indicates limit of detection. $P$ values were determined by comparing SKE MM-infected animals receiving IL6R antibody and SKE MM-infected animals receiving isotype control by ANOVA followed by Tukey's post hoc test. None of the differences are statistically significant.

in elaboration of TNFA by muscle fibers (53). In the context of inflammation, IL-1B and TNFA are potent inducers of IL- 6 production by skeletal muscle (51). The precise mechanism by which these molecules are elaborated following CHIKV infection of skeletal muscle remains undefined.

The 3- to 4-week-old mice used in these studies recapitulate many hallmarks of human disease, including muscle necrosis, myositis, and tenosynovitis (23). However, the inflammatory response to CHIKV in mice is not well-understood. Although molecular mediators of biphasic swelling in the inoculated foot are not known, $\mathrm{CD} 4^{+} \mathrm{T}$ cells are required for the second phase of swelling (39). We discovered that restriction of CHIKV replication in skeletal muscle results in diminished infiltration of $\mathrm{CD} 4^{+}$ $\mathrm{T}$ cells into the interosseous muscle at $7 \mathrm{dpi}$. Additionally, blockade of the IL-6 receptor significantly diminishes swelling during this phase, indicating that IL-6 receptor signaling is important for this disease manifestation. How IL- 6 receptor signaling and $\mathrm{CD} 4^{+} \mathrm{T}$ cells synergize to mediate this phenotype is unknown. IL-6 receptor expression is limited in mice, reaching detectable levels mainly on hepatocytes and some leukocytes (54). IL-6 induces chemotaxis of $\mathrm{T}$ cells in vitro, indicating a potential function in $\mathrm{T}$ cell homing to sites of CHIKV infection (55). Additionally, stimulation of naive T cells with IL-1B and IL-6 induces naive T cells to differentiate into TH17 cells (56). IL-17 elaborated by these cells is a mediator of inflammatory myopathy $(57,58)$. Stimulation of muscle cells with a combination of IL-1B, IL-17, and TNFA results in upregulation of MHC class I, which is directly cytotoxic to muscle cells (59). It is also possible that IL-6 functions in the activation of $\mathrm{CD} 4^{+} \mathrm{T}$ cells. IL-6 is a costimulatory molecule involved in $\mathrm{T}$ cell activation, expansion, and survival $(60,61)$. Additionally, costimulation of $\mathrm{CD}^{+}{ }^{+} \mathrm{T}$ cells with IL- 6 and TNFA is sufficient to activate these cells in an antigenindependent manner (62). Finally, while $\mathrm{CD}^{+} \mathrm{T}$ cells have a pathogenic role during CHIKV infection, it is also possible that $\mathrm{T}$ cells are required for viral clearance and that diminished infiltration by these cells caused by restriction of CHIKV replication in skeletal muscle could enhance persistence in musculoskeletal tissues at late times after inoculation.

While our studies have demonstrated that skeletal muscle cells are a critical infection site for development of severe musculoskeletal disease, there are a number of other important questions related to $\mathrm{CHIKV}$ pathogenesis that can be answered by coopting the host RNAi machinery. Cell culture-based susceptibility assays have implicated keratinocytes, fibroblasts, and skin-resident macrophages as potential primary sites of virus replication following deposition by the bite of an infected mosquito (10-12). The function of these cells in the amplification or dissemination of CHIKV following primary infection is unknown. Additionally, CHIKV is one of few arboviruses capable of achieving sufficiently high levels of viremia in humans to infect a naive mosquito following a blood meal (63). Because CHIKV is incapable of appreciable replication in leukocytes, serum viremia is likely the result of virus released from another cell type into the bloodstream (10). These gaps in knowledge could be addressed by engineering CHIKV strains restricted in other cell types through miRNA targeting.

Our study contributes to a better understanding of cellular and molecular determinants of disease development during CHIKV infection. These data also may enhance the development of CHIKV vaccines and targeted antivirals. To date, no CHIKV vaccines have achieved licensure, though a number of vaccine candidates are currently in clinical trials (64-66). Live-attenuated vaccines are often preferable, due to their capacity to elicit robust humoral and cellular immune responses and long-lived protection (67). One challenge in the development of live-attenuated vaccine candidates is to ensure an appropriate degree of attenuation to eliminate disease while allowing sufficient replication to induce a protective immune response. Addition of skeletal muscle cell-specific miRNA target sequences is an attractive method of attenuating CHIKV disease without affecting viral loads in tissues. Skeletal muscle cellspecific miRNA target sequences could potentially be incorporated 
into existing candidates to achieve ideal attenuation. Additionally, our studies identify IL- 6 receptor signaling as a critical mediator of CHIKV disease. Importantly, an IL-6 receptor antibody, tocilizumab, is approved for use in the treatment of rheumatoid arthritis (68). Our data indicate that this therapeutic could be used in the treatment of CHIKV disease. Overall, the findings reported here establish the foundation to understanding the contributions of discrete CHIKV-infected cell types to pathogenesis and potentially inform the development of effective vaccines and antivirals to limit the global burden of CHIKV disease.

\section{Methods}

Cells and viruses. Human osteosarcoma cells (U-2 OS; ATCC HTB-96) were maintained in McCoy's 5A medium (Gibco) supplemented to contain 10\% FBS (VWR). Baby hamster kidney cells (BHK-21; ATCC CCL-10) were maintained in alpha minimal essential medium ( $\alpha \mathrm{MEM}$; Gibco) supplemented to contain $10 \%$ FBS and 10\% tryptose phosphate. Vero81 cells (ATCC CCL-81) were maintained in $\alpha$ MEM supplemented to contain 5\% FBS. All cell maintenance medium was supplemented to contain $2 \mathrm{mM}$ L-glutamine (Gibco).

The WT CHIKV strain SL15649 infectious clone (pMH56) as well as a variant SL15649 infectious clone in which eGFP was introduced into the virus structural ORF (pMH75) were provided by Mark Heise (University of North Carolina at Chapel Hill). Skeletal muscle-restricted (SKE) and mismatch control (SKE MM) SL15649 strains were engineered to contain inserts designed in silico containing either 4 target sequences for skeletal muscle-specific miR-206 or 4 mismatch target sequences. These sequences were appended to a nucleotide sequence encoding 15 amino acids of the $2 \mathrm{~A}$ protease of foot-and-mouth disease virus (FMDV). Insert cassettes were synthesized by Genscript containing BssHII and ApaI restriction sites at the $5^{\prime}$ and $3^{\prime}$ termini, respectively. The pMH75 plasmid was digested with BssHII to release a 331bp fragment. The vector lacking this fragment was religated to produce a plasmid containing a single BssHII site (pMH75.1), which was then digested with BssHII and ApaI to remove the eGFP insert from the structural ORF. Inserts containing miR-target and mismatch sequences were digested from Genscript constructs using BssHII and ApaI and ligated into pMH75.1. The BssHII-BssHII fragment was then reinserted and screened for orientation by consensus sequencing.

Virus was recovered by linearization and in vitro amplification of infectious clone plasmids using the mMessage mMachine SP6 transcription kit (Ambion). BHK-21 cells were electroporated with in vitro-transcribed viral RNA using a Gene Pulser Xcell electroporator (Bio-Rad) and incubated at $37^{\circ} \mathrm{C}$ for 48 hours. Supernatants containing progeny virions were harvested, clarified by centrifugation at $1500 \mathrm{~g}$ at $4^{\circ} \mathrm{C}$ for 10 minutes to remove cell debris, and stored at $-80^{\circ} \mathrm{C}$. Titers of virus stocks were determined by plaque assay. All experiments using SL15649 and variant clones were conducted using biosafety level 3 conditions.

Viral plaque assays. Serial 10-fold dilutions of samples in virus dilution buffer (VDB; RPMI medium with 25 mM HEPES [Gibco] supplemented to contain $1 \% \mathrm{FBS}$ ) were adsorbed to Vero81 cells at $37^{\circ} \mathrm{C}$ for 1 hour. Monolayers were overlaid with $0.5 \%$ immunodiffusion agarose (VWR) in completed $\alpha \mathrm{MEM}$ and incubated at $37^{\circ} \mathrm{C}$ for 40 to 42 hours. Plaques were visualized following staining with neutral red (MilliporeSigma). Plaques were enumerated in duplicate and averaged to calculate PFU.
FFU assays. Serial 10-fold dilutions of samples in DMEM/F12 medium supplemented to contain 2\% FBS were adsorbed to Vero81 cells at $37^{\circ} \mathrm{C}$ for 2 hours. Monolayers were overlaid with $0.5 \%$ methylcellulose (MilliporeSigma) in medium and incubated at $37^{\circ} \mathrm{C}$ for 16 to 18 hours. Cells were fixed with $1 \%$ paraformaldehyde (PFA) in PBS at room temperature (RT) for 1 hour, washed 3 times with PBS, and permeabilized with perm/wash buffer (PBS supplemented to contain $0.1 \%$ saponin and $0.1 \%$ BSA) at RT for 5 minutes. Cells were incubated with CHIKV-specific monoclonal antibody CHK-11 (69) diluted to $500 \mathrm{ng} / \mathrm{mL}$ in perm/wash buffer at RT for 2 hours, washed 3 times with perm/wash buffer, incubated with a horseradish peroxidase-conjugated goat anti-mouse IgG secondary antibody (SouthernBiotech, 1030-05, 1:2000 dilution) at RT for 1 hour, and washed 3 times with perm/wash buffer. Foci were visualized following incubation with TrueBlue Substrate (Fisher) at RT for 10 minutes and enumerated using a CTL Biospot analyzer and Biospot software (Cellular Technology) to calculate FFU.

Assessment of CHIKV replication kinetics. U-2 OS cells were adsorbed with CHIKV strains diluted in VDB at an MOI of 0.01 PFU/ cell at $37^{\circ} \mathrm{C}$ for 1 hour. The viral inoculum was removed, cells were washed twice with PBS, and complete medium was added. Following incubation at $37^{\circ} \mathrm{C}$ for various intervals, $10 \%$ of the cell supernatant was collected and replaced with fresh complete medium. Viral titers in culture supernatants were determined by plaque assay.

Transfection of miRNA-mimic siRNAs. U-2 OS cells were transfected with $10 \mathrm{nM}$ of nonspecific siRNA (Luc), siRNA directed against the viral nsP1 gene, or a miR-206 mimic siRNA using Lipofectamine RNAiMAX (Invitrogen) diluted in serum-free OPTIMEM according to the manufacturer's instructions. Cells were incubated at $37^{\circ} \mathrm{C}$ for 12 hours and then adsorbed with CHIKV strains diluted in VDB at an MOI of $0.01 \mathrm{PFU} /$ cell at $37^{\circ} \mathrm{C}$ for 1 hour. The viral inoculum was removed, cells were washed twice with $\mathrm{PBS}$, and complete medium was added. After incubation at $37^{\circ} \mathrm{C}$ for various intervals, $10 \%$ of the cell supernatant was collected and replaced with fresh medium. Viral titers in culture supernatants were determined by plaque assay.

Mouse experiments. C57BL/6J mice were obtained from the Jackson Laboratory. All mouse infection studies were conducted in an animal biosafety level 3 laboratory. Three- to 4-week-old male mice were used for all studies. Mice were inoculated in the left rear footpad with $10 \mu \mathrm{L}$ containing either $10^{3}$ PFU of virus in diluent (PBS supplemented with $1 \%$ bovine calf serum [BCS]) or diluent alone (mock). Mice were weighed at 24-hour intervals and monitored for signs of disease. The area of the left rear footpad was determined prior to infection by measurement of footpad width and thickness with digital calipers and then at 24-hour intervals thereafter for either 7 or 14 days. For IL- 6 receptor blockade studies, mice were inoculated i.p. with $200 \mu \mathrm{g}$ of either $\alpha$ IL6R antibody (BioXCell, BE0047) or an IgG2b isotype control antibody (BioXCell, BE0090) diluted in PBS on days 0,3 , and 5 after inoculation with CHIKV. For experimental endpoints, mice were euthanized by exposure to isoflurane followed by thoracotomy. Blood was collected, and mice were perfused by intracardiac injection of PBS or 4\% PFA in PBS, depending on the experiment. PBS-perfused tissues were resected and homogenized using a MagNA Lyser (Roche) in either TRIzol reagent (Life Technologies) for RNA isolation or PBS supplemented to contain 1\% BCS for viral titer determination by FFU assay. 
RT-qPCR. RNA was isolated using a PureLink RNA minikit (Life Technologies), and $1 \mu \mathrm{g}$ RNA was reverse transcribed to cDNA using random primers (Thermo Fisher Scientific, 48190011) and the SuperScript IV first strand kit (Invitrogen). CHIKV sequencespecific forward primer (5'-TTTGCGTGCCACTCTGG-3'), reverse primer (5'-CGGGTCACCACAAAGTACAA-3'), and an internal TaqMan probe (5'-ACTTGCTTTGATCGCCTTGGTGAGA-3') were used to amplify and detect a region of the viral nsP2 gene. A standard curve was established from known samples containing 10 to $10^{8}$ genome copies of in vitro-transcribed CHIKV RNA. Purified RNA from BHK-21 cells was added to bring each standard sample to $1 \mu \mathrm{g}$ total RNA prior to reverse transcription in an identical manner to that used for experimental samples. Experimental samples were amplified concurrently with standards to quantify CHIKV genome copies/ $\mu \mathrm{g}$ RNA. Controls without template were processed in parallel. To determine host gene expression, cDNAs were subjected to qPCR analysis using TaqMan primer/probe sets specific for murine 18s rRna, Ifng, Il1b, Il6, Ip10, Mcp1, Mip1a, Rantes, and Tnfa (Thermo Fisher Scientific). Murine gene expression was normalized to 18s rRna values to control for differences in cDNA input. The relative fold induction of amplified mRNA relative to samples from mock-infected mice was determined using the Ct method (70).

Histopathological analysis. At defined times after inoculation, mice were euthanized, and PFA-perfused tissues were resected and fixed in $4 \%$ PFA in PBS at $4^{\circ} \mathrm{C}$ for at least 72 hours. Fixed tissue was embedded in paraffin, sectioned (5- $\mu \mathrm{m}$ thick), stained with $\mathrm{H} \& \mathrm{E}$, and visualized by light microscopy to assess histopathologic changes. Tissues resected at day 7 after inoculation were scored by a board-certified veterinary pathologist blinded to the conditions of the experiment for the presence, distribution, and severity of histopathological damage. For all tissue changes, the following scoring system was used: 0 , no lesions; 1 , mild, fewer than 5 areas of small clusters of leukocytes; 2, moderate, leukocytes forming larger clusters to thin tracts throughout the tissue, multiple sites/tissues affected; 3, severe, clusters and tracts of leukocytes coalescing into at least 1 large area that displaces/replaces tissue; 4 , markedly severe, leukocytes in aggregates sufficient to replace more than $40 \%$ of normal tissue. Immunohistochemical staining was conducted with tissue sections obtained from mice on day 7 after inoculation by incubation with antibodies directed against CD3 (Dako, A0452, 1:200 dilution) or CD4 (Abcam, ab183685, 1:1000 dilution) followed by incubation with a horseradish peroxidase-conjugated OmniMap anti-rabbit IgG secondary antibody (Ventana, 760-4311, ready to use). CD3 and CD4 signal in the interosseous muscle was quantified using ImageJ. Regions of interest containing interosseous muscle were defined from H\&E-stained serial sections of each foot. CD3- and CD4-stained images were separated into hematoxylin and DAB channels. DAB staining was quantified in the DAB channel for each interest region, and the intensity of CD3 and CD4 signal was calculated as the average of the staining intensity in each region weighted by its relative area.

In situ hybridization. At defined times after inoculation, mice were euthanized, and PBS-perfused tissues were resected and fixed in $4 \%$ PFA in PBS at $4^{\circ} \mathrm{C}$ for at least 72 hours. Tissue was washed 3 times in PBS at RT for 15 minutes, then 3 times in deionized water at RT for 15 minutes. Tissue was decalcified in 14\% EDTA and placed on an orbital shaker at RT. EDTA was replaced after 24 hours and at 72-hour intervals thereafter for 10 to 14 days until tissue was fully decalcified. Tissue was dehydrated, embedded in paraffin, and sectioned (5- $\mu \mathrm{m}$ thick). Viral RNA in situ hybridization was conducted using RNAscope 2.5 (Advanced Cell Diagnostics) according to the manufacturer's instructions. Tissue sections were incubated twice in xylene at RT for 5 minutes to remove paraffin, twice in 100\% ethanol at RT for 1 minute, once in hydrogen peroxide at RT for 10 minutes, and boiled in RNAscope Target Retrieval reagent (Advanced Cell Diagnostics) for 15 minutes. Slides were cooled to RT in deionized water and treated with RNAscope Protease Plus (Advanced Cell Diagnostics) at $40^{\circ} \mathrm{C}$ for 30 minutes before incubation with the hybridization probe. CHIKV RNA was detected using a probe (V-CHIKV-sp, 479501) designed by Advanced Cell Diagnostics. Tissues were counterstained with Gill's hematoxylin (MilliporeSigma) and visualized by light microscopy.

Statistics. All statistical tests were conducted using GraphPad Prism 7 software. Significant differences were detected using 2-tailed Student's $t$ test, Mann-Whitney test, or ANOVA with Tukey's post hoc test to correct for multiple comparisons. $P$ values of less than 0.05 were considered to be statistically significant. Descriptions of the specific statistical tests used for each experiment are provided in figure legends.

Study approval. All animal work reported here conforms to Public Health Service policy and was approved by the Institutional Animal Care and Use Committees at the University of Pittsburgh and University of Colorado School of Medicine.

\section{Author contributions}

AJL, MKM, LAS, TEM, and TSD designed research studies. AJL, MKM, NAM, BJD, and NM conducted experiments. AJL, MKM, NAM, BJD, and SAM acquired data. AJL, MKM, NAM, BJD, SAM, KR, LAS, TEM, and TSD analyzed data. AJL, MKM, LAS, TEM, and TSD wrote the manuscript.

\section{Acknowledgments}

This work was supported by Public Health Service awards T32 AI0 49820 (AJL), F32 AI122463 (MKM), T32 AR007543 (BJD), F31 AI147440 (NM), and R01 AI123348 (TSD and TEM). The authors thank Pavithra Aravamudhan and Danica Sutherland of the Dermody lab for critically reviewing the manuscript. We are grateful to members of the Dermody and Morrison laboratories for useful discussions during the conduct of these studies. Tissues were processed for histology and immunohistochemistry for CD3 and CD4 antigen at the histology core at the University of North Carolina at Chapel Hill. Histology slides were imaged by the University of Pittsburgh Biospecimen Core. The graphical abstract accompanying this manuscript was created with BioRender.com.

Address correspondence to: Terence S. Dermody, UPMC Children's Hospital of Pittsburgh, Administrative Office Building Suite 5300, 4401 Penn Avenue, Pittsburgh, Pennsylvania 15224, USA. Phone: 412.692.8071; Email: terence.dermody@chp.edu. Or to: Thomas E. Morrison, Department of Immunology and Microbiology, University of Colorado School of Medicine, 12800 East 19th Avenue, Mail Stop 8333, Aurora, Colorado 80045, USA. Phone: 303.724.4283; Email: thomas.morrison@cuanschutz.edu. 
1. Shin EC, Sung PS, Park SH. Immune responses and immunopathology in acute and chronic viral hepatitis. Nat Rev Immunol. 2016;16(8):509-523.

2. Jupp PG, McIntosh BM. Aedes furcifer and other mosquitoes as vectors of chikungunya virus at Mica, northeastern Transvaal, South Africa. J Am Mosq Control Assoc. 1990;6(3):415-420.

3. Ross RW. The Newala epidemic. III. The virus: isolation, pathogenic properties and relationship to the epidemic. J Hyg (Lond). 1956;54(2):177-191.

4. Diallo M, Thonnon J, Traore-Lamizana M, Fontenille D. Vectors of chikungunya virus in Senegal: current data and transmission cycles. Am J Trop Med Hyg. 1999;60(2):281-286.

5. Halstead SB. Reappearance of chikungunya, formerly called dengue, in the Americas. Emerging Infect Dis. 2015;21(4):557-561.

6. Zeller H, Van Bortel W, Sudre B. Chikungunya: Its History in Africa and Asia and Its Spread to New Regions in 2013-2014. J Infect Dis. 2016;214(suppl 5):S436-S440.

7. Leparc-Goffart I, Nougairede A, Cassadou S, Prat $\mathrm{C}$, de Lamballerie X. Chikungunya in the Americas. Lancet. 2014;383(9916):514.

8. Lemant $\mathrm{J}$, et al. Serious acute chikungunya virus infection requiring intensive care during the Reunion Island outbreak in 2005-2006. Crit Care Med.2008;36(9):2536-2541.

9. Borgherini $\mathrm{G}$, et al. Persistent arthralgia associated with chikungunya virus: a study of 88 adult patients on reunion island. Clin Infect Dis. 2008;47(4):469-475.

10. Sourisseau M, et al. Characterization of reemerging chikungunya virus. PLoS Pathog. 2007;3(6):e89.

11. Zhang R, et al. Mxra8 is a receptor for multiple arthritogenic alphaviruses. Nature. 2018;557(7706):570-574.

12. Puiprom $O$, et al. Characterization of chikungunya virus infection of a human keratinocyte cell line: role of mosquito salivary gland protein in suppressing the host immune response. Infect Genet Evol. 2013;17:210-215.

13. Couderc T, et al. A mouse model for Chikungunya: young age and inefficient type-I interferon signaling are risk factors for severe disease. PLoS Pathog. 2008;4(2):e29.

14. Nair S, Poddar S, Shimak RM, Diamond MS. Interferon regulatory factor 1 protects against chikungunya virus-induced immunopathology by restricting infection in muscle cells. J Virol. 2017;91(22):e01419-17.

15. Ozden S, et al. Human muscle satellite cells as targets of Chikungunya virus infection. PLoS ONE. 2007;2(6):e527.

16. Rohatgi A, et al. Infection of myofibers contributes to increased pathogenicity during infection with an epidemic strain of chikungunya virus. J Virol. 2014;88(5):2414-2425.

17. Hawman DW, et al. Mutations in the E2 glycoprotein and the 3' untranslated region enhance chikungunya virus virulence in mice. J Virol. 2017;91(20):e00816-17.

18. Paquet $\mathrm{C}$, et al. Chikungunya outbreak in Reunion: epidemiology and surveillance, 2005 to early January 2006. Euro Surveill. 2006;11(2):E060202.3.

19. Chow A, et al. Persistent arthralgia induced by chikungunya virus infection is associated with interleukin-6 and granulocyte macrophage colony-stimulating factor. J Infect Dis. 2011;203(2):149-157.

20. Nkoghe D, et al. Clinical forms of chikungunya in Gabon, 2010. PLoS Negl Trop Dis. 2012;6(2):e1517.

21. Staikowsky F, et al. Prospective study of chikungunya virus acute infection in the Island of $\mathrm{La}$ Réunion during the 2005-2006 outbreak. PLoS ONE. 2009;4(10):e7603.

22. Rezza G, et al. Infection with chikungunya virus in Italy: an outbreak in a temperate region. Lancet. 2007;370(9602):1840-1846.

23. Morrison TE, et al. A mouse model of chikungunya virus-induced musculoskeletal inflammatory disease: evidence of arthritis, tenosynovitis, myositis, and persistence. Am J Pathol. 2011;178(1):32-40.

24. Martinez J, Patkaniowska A, Urlaub H, Lührmann R, Tuschl T. Single-stranded antisense siRNAs guide target RNA cleavage in RNAi. Cell. 2002;110(5):563-574.

25. Lagos-Quintana M, Rauhut R, Yalcin A, Meyer J, Lendeckel W, Tuschl T. Identification of tissue-specific microRNAs from mouse. Curr Biol. 2002;12(9):735-739.

26. Landgraf $\mathrm{P}$, et al. A mammalian microRNA expression atlas based on small RNA library sequencing. Cell.2007;129(7):1401-1414.

27. Guo Z, Maki M, Ding R, Yang Y, Zhang B, Xiong L. Genome-wide survey of tissue-specific microRNA and transcription factor regulatory networks in 12 tissues. Sci Rep. 2014;4:5150.

28. Ylösmäki E, Martikainen M, Hinkkanen A, Saksela K. Attenuation of Semliki Forest virus neurovirulence by microRNA-mediated detargeting. JVirol. 2013;87(1):335-344.

29. Soumahoro MK, et al. Impact of chikungunya virus infection on health status and quality of life: a retrospective cohort study. PLOS ONE. 2009;4(11):e7800.

30. Staikowsky F, et al. Retrospective survey of chikungunya disease in Réunion Island hospital staff. Epidemiol Infect. 2008;136(2):196-206.

31. Schilte $\mathrm{C}$, et al. Chikungunya virus-associated long-term arthralgia: a 36-month prospective longitudinal study. PLoS Negl Trop Dis. 2013;7(3):e2137.

32. Horak M, Novak J, Bienertova-Vasku J. Muscle-specific microRNAs in skeletal muscle development. Dev Biol. 2016;410(1):1-13.

33. Anderson C, Catoe H, Werner R. MIR-206 regulates connexin 43 expression during skeletal muscle development. Nucleic Acids Res. 2006;34(20):5863-5871.

34. Chen JF, et al. microRNA-1 and microRNA-206 regulate skeletal muscle satellite cell proliferation and differentiation by repressing Pax7. JCell Biol. 2010;190(5):867-879.

35. Kelly EJ, Hadac EM, Greiner S, Russell SJ. Engineering microRNA responsiveness to decrease virus pathogenicity. Nat Med.2008;14(11):1278-1283.

36. He F, et al. Coxsackievirus B3 engineered to contain microRNA targets for muscle-specific microRNAs displays attenuated cardiotropic virulence in mice. J Virol. 2015;89(2):908-916.

37. Sun C, Gardner CL, Watson AM, Ryman KD,
Klimstra WB. Stable, high-level expression of reporter proteins from improved alphavirus expression vectors to track replication and dissemination during encephalitic and arthritogenic disease. JVirol. 2014;88(4):2035-2046.

38. McCarthy MK, et al. Chikungunya virus impairs draining lymph node function by inhibiting HEV-mediated lymphocyte recruitment. JCI Insight. 2018;3(13):121100.

39. Teo TH, et al. A pathogenic role for $\mathrm{CD} 4+\mathrm{T}$ cells during chikungunya virus infection in mice. JImmunol. 2013;190(1):259-269.

40. Gardner J, et al. Chikungunya virus arthritis in adult wild-type mice. JVirol. 2010;84(16):8021-8032.

41. Ng LF, et al. IL-1beta, IL-6, and RANTES as biomarkers of chikungunya severity. PLOS ONE. 2009;4(1):e4261.

42. Venugopalan A, Ghorpade RP, Chopra A. Cytokines in acute chikungunya. PLoS ONE. 2014;9(10):e111305.

43. Morrison TE, Whitmore AC, Shabman RS, Lidbury BA, Mahalingam S, Heise MT. Characterization of Ross River virus tropism and virus-induced inflammation in a mouse model of viral arthritis and myositis. JVirol. 2006;80(2):737-749.

44. Sane J, et al. Prolonged myalgia in Sindbis virus infection: case description and in vitro infection of myotubes and myoblasts. J Infect Dis. 2012;206(3):407-414.

45. Crum-Cianflone NF. Nonbacterial myositis. Curr Infect Dis Rep. 2010;12(5):374-382.

46. Agyeman P, Duppenthaler A, Heininger U, Aebi C. Influenza-associated myositis in children. Infection. 2004;32(4):199-203.

47. Singh U, Scheld WM. Infectious etiologies of rhabdomyolysis: three case reports and review. Clin Infect Dis. 1996;22(4):642-649.

48. Ishiuchi Y, et al. Skeletal muscle cell contraction reduces a novel myokine, chemokine ( $\mathrm{C}-\mathrm{X}-\mathrm{C}$ motif) ligand 10 (CXCL10): potential roles in exercise-regulated angiogenesis. Biosci Biotechnol Biochem. 2018;82(1):97-105.

49. Karalaki M, Fili S, Philippou A, Koutsilieris M. Muscle regeneration: cellular and molecular events. In Vivo. 2009;23(5):779-796.

50. Keller P, et al. Interleukin- 6 production by contracting human skeletal muscle: autocrine regulation by IL-6. Biochem Biophys Res Commun. 2003;310(2):550-554.

51. Muñoz-Cánoves P, Scheele C, Pedersen BK, Serrano AL. Interleukin-6 myokine signaling in skeletal muscle: a double-edged sword? FEBS J. 2013;280(17):4131-4148.

52. Podbregar M, Lainscak M, Prelovsek O, Mars T. Cytokine response of cultured skeletal muscle cells stimulated with proinflammatory factors depends on differentiation stage. ScientificWorldJournal. 2013;2013:617170.

53. Collins RA, Grounds MD. The role of tumor necrosis factor-alpha (TNF-alpha) in skeletal muscle regeneration. Studies in TNF-alpha(-/-) and TNF-alpha(-/-)/LT-alpha(-/-) mice. J Histochem Cytochem. 2001;49(8):989-1001.

54. Saito M, Yoshida K, Hibi M, Taga T, Kishimoto T. Molecular cloning of a murine IL-6 receptor-associated signal transducer, gp130, and its regulated expression in vivo. J Immunol. 1992;148(12):4066-4071 
55. Weissenbach M, et al. Interleukin-6 is a direct mediator of T cell migration. Eur J Immunol. 2004;34(10):2895-2906.

56. Dienz O, Rincon M. The effects of IL- 6 on CD4 T cell responses. Clin Immunol. 2009;130(1):27-33.

57. Tournadre A, Miossec P. Interleukin-17 in inflammatory myopathies. Curr Rheumatol Rep. 2012;14(3):252-256.

58. Chevrel G, Page G, Granet C, Streichenberger N, Varennes A, Miossec P. Interleukin-17 increases the effects of IL-1 beta on muscle cells: arguments for the role of $\mathrm{T}$ cells in the pathogenesis of myositis. J Neuroimmunol. 2003;137(1-2):125-133.

59. Nagaraju K, et al. Activation of the endoplasmic reticulum stress response in autoimmune myositis: potential role in muscle fiber damage and dysfunction. Arthritis Rheum. 2005;52(6):1824-1835.

60. Lotz M, et al. B cell stimulating factor 2/interleukin 6 is a costimulant for human thymocytes and $\mathrm{T}$ lymphocytes. J Exp Med.1988;167(3):1253-1258.

61. Rochman I, Paul WE, Ben-Sasson SZ. IL-6 increases primed cell expansion and survival. JImmunol. 2005;174(8):4761-4767.

62. Unutmaz D, Pileri P, Abrignani S. Antigen-independent activation of naive and memory resting T cells by a cytokine combination. J Exp Med. 1994;180(3):1159-1164.

63. Weaver SC. Evolutionary influences in arboviral disease. Curr Top Microbiol Immunol. 2006;299:285-314.

64. Chang LJ, et al. Safety and tolerability of chikungunya virus-like particle vaccine in healthy adults: a phase 1 dose-escalation trial. Lancet. 2014;384(9959):2046-2052.

65. Ramsauer K, et al. Immunogenicity, safety, and tolerability of a recombinant measles-virus-based chikungunya vaccine: a randomised, double-blind, placebo-controlled, active-comparator, first-in-man trial. Lancet Infect Dis. 2015;15(5):519-527.

66. Plante KS, Rossi SL, Bergren NA, Seymour RL, Weaver SC. Extended preclinical safety, efficacy and stability testing of a live-attenuated chikungunya vaccine candidate. PLoS Negl Trop Dis. 2015;9(9):e0004007.

67. Lauring AS, Jones JO, Andino R. Rationalizing the development of live attenuated virus vaccines. Nat Biotechnol. 2010;28(6):573-579.

68. Scott LJ. Tocilizumab: A review in rheumatoid arthritis. Drugs. 2017;77(17):1865-1879.

69. Pal P, et al. Development of a highly protective combination monoclonal antibody therapy against chikungunya virus. PLoS Pathog. 2013;9(4):e1003312.

70. Livak KJ, Schmittgen TD. Analysis of relative gene expression data using real-time quantitative PCR and the 2(-Delta Delta C(T)) Method. Methods. 2001;25(4):402-408. 